\title{
4 Bewertung des Patientennutzens
}

Der Staat, Behörden oder öffentlich-rechtliche Institutionen als Sachwalter der Bürger, Versicherten oder Patienten entscheiden über die Ausgestaltung der Versorgung und die Leistungen im Rahmen der GKV. Hierzu bedarf es der Bewertung und Evaluation von Leistungsangeboten und der Prognose von Nachfrage und Akzeptanz der zur Verfügung stehenden Alternativen. Wenn ein Anbieter ein Verfahren, ein Produkt oder eine Technologie im deutschen Gesundheitssystem einbringen will, geht es primär um die Frage: „Was hat der Patient davon?“.

In Studien konnte gezeigt werden, dass sich Patientenpräferenzen und das Expertenurteil bezüglich des Patientennnutzens zum Teil erheblich unterscheiden. ${ }^{[119,}{ }^{120]}$ Daher sollten Informationen über die Einschätzung und Sichtweise der Patienten als weitere Entscheidungshilfe herangezogen werden.

\subsection{Herausforderungen multikriterieller Entscheidungsprobleme}

Im vorangegangenen Kapitel wurden die methodischen Herausforderungen der Erfolgsmessung im Kontext der evidenzbasierten Medizin diskutiert. Die praktische Implementierung der evidenzbasierten Medizin, die Umsetzung höchster Anforderungen an das Studiendesign und eine hohe Ergebnissicherheit garantieren die bestmögliche Information über kausal begründete Effekte einer Intervention im Vergleich mit dem Status Quo oder Placebo. Die Messung der klinischen und nicht-klinischen Effekte ist eine notwendige Bedin-

gung für die Entscheidung über den Einsatz und die Erstattungsfähigkeit von 
MP. Es ist eine notwendige, jedoch keine hinreichende Bedingung. Eine rationale Entscheidung bzw. Auswahl der optimalen Alternative, ist mit diesen Informationen nicht möglich. Unklar bleibt, mit welchen Werturteilen das Maß des Gesamtnutzens bzw. das Ausmaß des Nutzens im Vergleich mit einer alternativen Verwendung quantifiziert werden soll. Werturteile der Experten und Präferenzen der Betroffenen müssen bei der Entscheidung systematisch und transparent berücksichtigt werden. Es kann von einem erheblichen Handlungsbedarf ausgegangen werden, da eine konsistente systematische Nutzenbewertung für Untersuchungs- und Behandlungsmethoden mit MP hoher Klasse eine wesentliche Voraussetzung für konsistente und valide Entscheidungen über die Erstattungsfähigkeit ist.

Das IQWiC Methodenpapier definiert das „Maß des Cesamtnutzens“ als „eine Aggregierung der Bewertung von Nutzen und Schaden in einer Größe, wobei unterschiedliche patientenrelevante Endpunkte zu einem einzigen Maß zusammengefasst werden“..[1] Die Operationalisierung erfolgt über die Abwägung und Aggregation von positiven und negativen Effekten einer Intervention. Bei der Dokumentation des Gesamtnutzens muss zu Beginn bestimmt werden, welche Zielkriterien aus den Nutzendimensionen abgeleitet werden können. Konkret: Welche Zielkriterien oder Endpunkte werden bei der Bestimmung des Nutzens berücksichtigt? Die Ableitung von Zielkriterien bzw. patientenrelevanten Endpunkten ist abhängig von der zugrundeliegenden Definition des Nutzens (s. hierzu die Ausführungen zu „Zielkriterien, Zielgrößen oder Nutzendimensionen" in Kap. 3.2).

Das IQWiC Methodenpapier unterscheidet zwei Ansätze zur Dokumentation eines Maßes des Gesamtnutzens: (a) den "QALY als Maß des Gesamtnutzens“ und (b) die „Erhebung von Präferenzen zur Erstellung eines Maßes des Gesamtnutzens". Im Kern geht es bei dieser Unterscheidung darum, dass bei der ersten Alternative patientenberichtete subjektive Angaben zur Lebensqualität bzw. dem Gesundheitszustand (nicht-klinische Endpunkte) gewichtet werden, um dann einen Nutzenwert abzuleiten. Das zweite Maß basiert auf einem breiteren Ansatz (hier bezeichnet mit „Multikriterielle Entscheidungsfindung") und gewichtet klinische und nicht-klinische Endpunkte auf Grundlage wissenschaftlich abgeleiteter Präferenzen. Beide Verfahren haben gemein, dass nur mit Hilfe der Gewichtung der realisierten Entscheidungskriterien ein Nutzenwert als Maß des Gesamtnutzens abgeleitet werden kann.

Versteht man die Nutzenbewertung als „Prozess der Evaluation medizinischer Interventionen hinsichtlich ihrer kausal begründeten positiven und negativen Effekte im Vergleich mit einer klar definierten anderen Therapie, einem Placebo (oder einer andersartigen Scheinbehandlung) oder keiner Behandlung" ${ }^{[1]}$, dann müssen die positiven und negativen Effekte (Nutzen- und Schadensaspekte) zunächst für jede einzelne Therapiealternative oder jede Methode mit MP gegeneinander abgewogen werden. Bei Vorliegen multipler Endpunkte oder Entscheidungskriterien ist eine Gewichtung der Entscheidungskriterien 
die wesentliche Voraussetzung für die gemeinsame Würdigung der endpunktbezogenen Nutzen- und Schadensaspekte (unabhängig davon, ob es sich um „subjektive“ Gesundheitszustände oder „objektive“ klinische Effekte handelt). Eine implizite Gewichtung durch intransparente Werturteile widerspricht der Forderung nach Transparenz. Die Gewichtung der Zielkriterien erfolgt dann unabhängig von den Bedürfnissen der Konsumenten. Dieses Vorgehen deckt sich weder mit dem allgemeinen Gerechtigkeitsempfinden noch mit den Wertmaßstäben der Bevölkerung. Diese Argumentation erfährt Unterstützung durch das IQWiC selbst. Es wird durch das IQWiC darauf hingewiesen, dass die Präferenzen der (potenziellen) Patienten bei der Nutzenbewertung eine Rolle spielen und bei der Nutzenbewertung berücksichtigt werden sollten. ${ }^{[22]}$

\subsection{Multikriterielle Entscheidungsanalyse als methodische Basis}

Da die aktuellen Methoden des HTA für die Bewertung von NUB nur bedingt anwendbar sind, könnte die multikriterielle Entscheidungsanalyse als Alternative und als ein Instrumentarium angesehen werden, einige der Beschränkungen der herkömmlichen HTA zu überwinden. ${ }^{[28]}$ Die niederländische HTAAgentur untersucht beispielsweise, ob Methoden des MCDA in die Bewertung von MP integriert werden sollten, um die Grenzen der klassischen KostenNutzen-Analyse zu überwinden. ${ }^{[22,}$, 123] Das IQWiC nennt Methoden der MCDA und der Präferenzmessung als eine zu nutzende Methode, wenn „ein Maß des Gesamtnutzens zum Vergleich von Interventionen erhoben werden soll“" (IQWiG Methodenpapier 4.2, S. 85 ff. $)^{[1]}$

Als methodische Basis und Rahmenkonstrukt für alle Bewertungsprozesse kann die multikriterielle Entscheidungsanalyse (Multi-Criteria Decision Analysis, MCDA) dienen. Unter dem Oberbegriff der „Multikriteriellen Entscheidungsfindung "werden dabei verschiedene analytische Methoden zur Berücksichtigung multipler Entscheidungskriterien zusammengefasst. [26, 27]

Allen Ansätzen ist gemein, dass der Patientennutzen (Gesamtnutzen) als multidimensionales Konstrukt angesehen wird, welcher nur unter Einbeziehung der betroffenen Patienten bewertet werden kann. Der wahrgenommene oder erwartete Nutzen aus Sicht des Patienten ist die Basis für Präferenzen. Aus diesem Grund können auf Grundlage dokumentierter Präferenzen Rückschlüsse auf den Nutzen einer Bewertungsalternative gezogen werden. ${ }^{[24]}$ Als solches kann die Integration von MCDA in Bewertungen als Qualitätsgarantie im Sinne der Patientenperspektive angesehen werden .

Insbesondere bei der Nutzenbewertung spielt die Perspektive der Patienten bislang nur eine sehr untergeordnete Rolle. ${ }^{[2]}$ Allerdings sollten die Rechte der Patienten gerade vor dem Hintergrund innovativer Behandlungsmethoden berücksichtigt werden. Hier ist die Frage, ob Patienten die Chance erhalten (sollten), über die Risikoprofile einzelner Therapiealternativen mitzubestim- 
men. Die Anerkennung von Patientenautonomie bedeutet in diesem Fall, dass den Patienten die Gelegenheit gegeben wird, nach ausführlicher Information und in Abhängigkeit der Indikation, ihre individuellen Risikopräferenzen bezüglich verschiedener Therapien zum Ausdruck zu bringen. ${ }^{[25]}$

Das gesteigerte Interesse an der MCDA spiegelt sich in einem verstärkten Einsatz der MCDA im Gesundheitswesen wider, wie jüngste systematische Literaturübersichten zeigen. ${ }^{[26,}{ }^{127]}$ Die verschiedenen Methoden bzw. Techniken der multikriteriellen Entscheidungsanalyse wurden im Rahmen des Operations Research, des Marketings und der Entscheidungsanalyse entwickelt. ${ }^{[28]}$ MCDA repräsentiert einen praktischen Weg, um Präferenzen für Alternativen im Gesundheitswesen zu berücksichtigen, die unter Alltagsbedingungen oder bei traditionellen Gremien- oder Gruppenentscheidungen nur schwierig (wenn nicht unmöglich) zu beobachten sind. ${ }^{[22]]}$ MCDA kann ebenso genutzt werden, um geeignete Kriterien bei Entscheidungsfindungen im Cesundheitswesen zu definieren, indem gemessen wird, welchen Nutzen Patienten den verschiedenen Kriterien beimessen, die die Entscheidungen im Gesundheitswesen beeinflussen. ${ }^{[2]}$ Damit kann die MCDA im Gesundheitswesen grundsätzlich sowohl vorwärts als auch rückwärts gerichtet zum Einsatz kommen: bei der Zulassung und der Evaluation.

Im Fokus stehen Entscheidungen, die entweder durch in Konflikt stehende Zielkriterien oder aufgrund hoher Ungewissheit problematisch sind. Die entscheidungstheoretisch fundierte MCDA trägt dazu bei, einen komplexen Entscheidungsprozess zu strukturieren.

Grundsätzlich besteht der Prozess der meisten Methoden der multikriteriellen Entscheidungsanalyse aus acht aufeinander aufbauenden Schritten ${ }^{[130]}$ :

- Definition der Ziele und des Entscheidungsproblems: Im ersten Schritt wird das übergeordnete Handlungsziel formuliert, um den Entscheidungsgegenstand vollständig und eindeutig zu erfassen.

- Bestimmung der Alternativen: Alternativen beschreiben Wahlmöglichkeiten, die zur Lösung des Entscheidungsproblems zur Auswahl stehen. Dies könnten beispielsweise verschiedene MP oder Untersuchungs- und Behandlungsmethoden sein.

- Festlegung der Zielkriterien: Die Kriterien, häufig auch als Eigenschaften oder Attribute bezeichnet, stellen die entscheidungsrelevanten Einflussfaktoren des Entscheidungsproblems dar. Anhand dieser Kriterien kann die Zielerreichung gemessen werden. Bei der Bewertung von NUB können mehrere Zielkriterien formuliert (z.B. Mortalität, Morbidität, Lebensqualität, Patientenzufriedenheit) und durch Nutzendimensionen abgebildet (z.B. Verbesserung des Gesundheitszustandes, Verkürzung der Krankheitsdauer, Verlängerung der Lebensdauer, Verbesserung der Lebensqualität oder Verbesserung der Anwendung bzw. Prozessverbesserung) werden. 
- Messung der Zielerreichung: Hier werden alle definierten Kriterien alternativen-spezifisch gemessen (s. hierzu Kap. 3). Zielsetzung ist die Darlegung eines kausalen Zusammenhangs zwischen den NUB und positiven oder negativen Effekten. Klinische Studien sind die Basis der Nutzenbewertung medizinischer Methoden. Klinische und nicht-klinische Zielgrößen werden mit einem geeigneten Studiendesign gemessen. Ziel ist die Generierung von Daten zum patientenrelevanten (Zusatz-)Nutzen von NUB. Qualitativ hochwertige Studien müssen eine ausreichende Ergebnissicherheit garantieren.

- Bewertung der Zielerreichungsgrade: Dieser auch als Scoring bezeichnete Schritt ist ein Instrument zur mehrdimensionalen Bewertung von Alternativen. Es werden dabei quantitative und qualitative Kriterien einbezogen. Für den Vergleich der Zielerreichung auf unterschiedlichen Bewertungskriterien ist eine Bewertungsskala für alle Kriterien festzulegen (z.B. 1-10 Punkte). Bewertet wird, inwieweit einzelne Interventionen die gesetzten Ziele erreichen (Zielerreichungsgrad). Die Intervention mit dem höchsten Zielerreichungsgrad erreicht den höchsten Punktwert. Das Scoring erfolgt, um die Zielerreichung für unterschiedliche Zielkriterien vergleichbar zu machen.

- Gewichtung der Zielkriterien: Die Gewichtung drückt aus, wie stark die Kriterien bei der Bewertung berücksichtigt werden. Bewertungskriterien lassen sich hinsichtlich der Relevanz für den Patienten gewichten. Hierbei können unterschiedliche Gewichtungsmethoden zum Einsatz kommen.

- Aggregation des Gesamtnutzens: Nach der Punktbewertung und Gewichtung können Werte für den Teilnutzen der definierten Kriterien berechnet werden. Diese ergeben sich aus den gewichteten Zielerreichungsgraden. Abschließend erfolgt die Aggregation des Gesamtnutzens. Ziel ist es, die Ergebnisse vergleichbar zu machen. Dabei wird für jede Alternative ein Gesamtnutzenwert ermittelt, der sich aus den zuvor ermittelten Teilnutzenwerten zusammensetzt.

- Rangreihung der Alternativen: Aus diesen Werten resultiert das Ranking, das auf der Höhe der Werte basiert. Bei Entscheidungen im Gesundheitswesen sollen nicht nur mehrere Zielsetzungen berücksichtigt, sondern auch mehrere Alternativen miteinander verglichen werden. Am Ende steht ein Faktor bzw. eine Maßzahl für den Gesamtnutzen, der systematisch über alle Alternativen anwendbar ist.

Dieser letzte Schritt bietet dabei die Möglichkeit einer Klassifizierung und eines Rankings der Interventionen/Kriterien innerhalb des gewählten Versorgungskontexts oder einer bestimmten Indikation (League table). ${ }^{[131]}$ In Zukunft sollten diese Informationen genutzt werden, um Entscheidungsträger auf klinischer und regulatorischer Ebene über klinische Faktoren zu informieren. ${ }^{[21]}$ 


\subsection{Forderung nach der Aggregation von Nutzen und Schaden}

Kausal begründete klinische Effekte können einen Nutzen oder Schaden für den Patienten darstellen. Damit Methoden mit MP verglichen werden können, muss der Gesamtnutzen als eindimensionale Größe bestimmt werden. Die Aggregation des Gesamtnutzens erfordert die Abwägung von Nutzen und Schaden. Nutzen im ökonomischen Sinne resultiert aus der Bewertung der erwünschten und unerwünschten klinischen Effekte. Der Nutzen entsteht beim Konsumenten einer Leistung. Damit erfolgt die Bewertung der Effekte in der Regel aus der Perspektive des Patienten.

Nutzen kann durch Dritte nicht direkt beobachtet werden. Die ordinale Nutzentheorie definiert den Nutzen als einen nicht direkt messbaren Sachverhalt, der sich aus individuellen Wahlhandlungen ableiten lässt. Die Präferenz beschreibt die Vorziehenswürdigkeit einer Alternative über eine oder mehrere andere Alternativen. Der Patientennutzen einer Intervention ist damit für Dritte primär theoretischer Natur und muss aus messbaren Sachverhalten (Indikatoren) abgeleitet werden. Wahlhandlungen können beobachtet werden . Wahlhandlungen werden ausgelöst durch die Eigenschaften einer Intervention. Die Eigenschaften können durch die Ausprägungen der Zielkriterien beschrieben werden. In diesem Sinne spricht man auch vom latenten Konstrukt, d.h. für die Operationalisierung muss ein latentes Variablenmodell erstellt werden. Dabei sind die gemessenen klinischen Effekte vom daraus resultierenden Patientennutzen zu unterscheiden. Indirekt kann der Nutzen eines Individuums aufgrund von Wahlhandlungen beobachtet werden, da davon ausgegangen werden kann, dass ein Individuum stets diejenige Alternative wählt, die den persönlichen Nutzen maximiert. Nettonutzen ist dabei als das positive Ergebnis der vergleichenden Abwägung von Nutzen und Schaden einer Intervention hinsichtlich der Zielkriterien bei der Behandlung von Patienten bzw. Patientengruppen definiert. Der zusätzliche Nutzen im Vergleich ist das positive Ergebnis der vergleichenden Abwägung von Interventionen hinsichtlich des bewerteten Nettonutzens. Folgende Aspekte können dabei im Mittelpunkt stehen: Relevanz der Zielkriterien, Werturteile bzw. Präferenzen im Hinblick auf die Gewichtung und Vergleichbarkeit der Effekte sowie Annahmen über die Aggregation multipler Zielkriterien.

Für den Vergleich von Alternativen ist es jedoch notwendig, dass ein eindimensionales Maß des Patientennutzens aus den gemessenen Effekten aggregiert werden kann. Hierzu bedarf es gewichteter Effekte. Maßstab für die Gewichtung sind die Präferenzen der Patienten. In diesem Kontext ist die einheitliche Definition und Ableitung der Patientenpräferenzen eine wesentliche Voraussetzung für die vergleichende Bewertung und damit für die Wahlentscheidung zwischen MP oder Interventionen. Formal gesehen lassen sich drei Schwerpunkte für die Phase des Bewertens eines neuen theoretisch-wissenschaftlichen Konzepts von NUB mit MP hoher Klassen ableiten: 
- Abwägung von Nutzen und Schaden: Nachdem die Effekte hinsichtlich der identifizierten Zielkriterien gemessen wurden, müssen der Nutzen (erwartetes Nutzenpotenzial) und der Schaden (erwartetes Schadenspotenzial) abgewogen werden. Erfolgt die Bewertung über Dritte bzw. Experten, dann ermöglichen explizite Werturteile die transparente Abwägung von Nutzen (erwünschten Effekten) und Schaden (unerwünschten Effekten). Zielsetzung ist die Bereitstellung von Daten, welche die Entscheidungsträger über Werturteile von Versicherten, Patienten und der Bevölkerung informieren.

- Perspektive der Nutzenbewertung: Der G-BA bewertet den Nutzen oder das Potenzial eines MP. Unklar bleibt, welche Perspektive (Patient, Versicherter oder Bürger) handlungsleitend in der Nutzenbewertung von neuen NUB mit MP hoher Klassen sein wird. Diskutiert werden Optionen (Klinischer Nutzen, Patientennutzen, Anwendernutzen) und Implikationen unterschiedlicher Nutzenbegriffe.

- Aggregation multipler klinischer oder nicht-klinischer Endpunkte: Multiple Zielkriterien erhöhen die Komplexität bei der Entscheidung. Zielkriterien mit eventuell divergierenden Effekten müssen vergleichbar gemacht werden. Der Nachweis eines höheren Gesamtnutzens erfolgt in Relation zum Komparator. Diskutiert werden die Vergleichbarkeit von klinischen Effekten und deren Aggregation zu einem Gesamtmaß des Nutzens.

Ein Nutzen wird folglich aus der (erwarteten) Wirksamkeit abgeleitet, entspricht aber nicht den Wirksamkeitsparametern. Bei der Ableitung des Nutzens aus den Effektgrößen sind deshalb mehrere Wertentscheidungen notwendig. Unklar ist, in welchem funktionalen Zusammenhang ein Effekt einen Nutzen beim Patienten begründet. Werden mehrere Endpunkte für die Nutzenbewertung herangezogen, müssen Nutzen und Schaden abgewogen werden. Präferenzgewichte sollten zuverlässig und korrekt die relative Wichtigkeit der Endpunkte abbilden. Die relative Wichtigkeit eines Zielkriteriums bedeutet, dass sich die Bedeutung eines Endpunktes immer in Relation zur Bedeutung alternativer Zielkriterien bemisst. Das Bewerten von Endpunkten und Alternativen ist dabei Gegenstand der Ökonomie und Statistik, insbesondere der Entscheidungstheorie. Zielsetzung ist die systematische Zusammenfassung aller Informationen über die Effekte in einem (eindimensionalen) Nutzenmaß (Aggregation der Zielerreichungsgrade).

Ergebnis der Bewertung ist die Bereitstellung von Informationen, d.h. indikationsspezifischer oder indikationsübergreifender Kennzahlen, als Grundlage für eine Entscheidung. Der rationale Vergleich von Interventionen erfolgt anhand dieses eindimensionalen Nutzenmaßes und ist eine notwendige Bedingung für die Ermittlung des Nutzens einer Intervention im Vergleich mit einer Vergleichsintervention.

Bei der Bewertung von MP stellt sich insbesondere die Frage nach der Definition des „Nutzens“. Wird die ökonomische Betrachtung außer Acht gelassen, 
so lässt sich eine abwägende Bilanzierung von Nutzen und Risiko aus patientenorientierter Sicht nicht nachvollziehen. Die Vor- und Nachteile einer Methode oder eines MP sind nicht eindeutig definiert und eine wirtschaftliche Versorgung ist nicht möglich.

\subsection{Diskussion der Bewertung multipler Endpunkte}

Dieses Kapitel befasst sich nicht mit den Methoden der Messung klinischer und nicht-klinischer Erfolgsparameter, der Begründung unterschiedlicher Studiendesigns, biometrischen Anforderungen oder der sachgerechten Umsetzung der EbM. Dieses Kapitel fokussiert einzig und allein auf die Bewertung der gemessenen klinischen und nicht-klinischen Effekte. Damit konzentrieren sich die Ausführungen auf die Bewertung der gemessenen Effekte als Kern und notwendige Bedingung für die Nutzenbewertung. Es geht darum, wie die Multidimensionalität des Patientennutzens (Mortalität, Morbidität, Lebensqualität) bei der Entscheidung berücksichtigt werden kann. Zudem geht es darum, wie die patientenrelevanten Endpunkte bei der Abwägung des Nutzen und Schadens, der Aggregation eines Maßes des Gesamtnutzens oder des Ausmaßes des zusätzlichen Nutzens im Vergleich mit weiteren Alternativen in die Entscheidung eingehen.

Es bleibt bis heute bei der Entscheidung über MP offen, welche wissenschaftlichen Methoden und Instrumente zur Dokumentation der Relevanz oder Wichtigkeit eines Zielkriteriums systematisch herangezogen werden. Bei einem unklaren Nutzenbegriff bleibt ungelöst, welche Zielkriterien bei der Abwägung von Nutzen und Schaden zur Bestimmung des zusätzlichen Nutzens oder Gesamtnutzens eingehen. Die gemeinsame Würdigung von Nutzen und Schaden verlangt einen transparenten Prozess, der die Wertentscheidungen, d.h. die Gewichtung der positiven und negativen Effekte, offen legt. Wertentscheidungen der Abwägung sollten nicht Gegenstand „subjektiv geprägter und damit häufig verzerrter" Bewertungen sein. ${ }^{[1]}$ Ohne wissenschaftlich belastbare Aussagen zu Wertmaßstäben und Präferenzen zu den patientenrelevanten Endpunkten ist eine Abwägung von Nutzen und Schaden nicht möglich.

Angenommen, man könnte davon ausgehen, dass die Messung mehrerer patientenrelevanter Endpunkte zu 100\% die Wirksamkeit einer Intervention A im Vergleich zu einer Intervention B wiedergibt, dann kann auf Basis dieses Wissens keine Aussage über den Nutzen, den Gesamtnutzen, den Nettonutzen oder den zusätzlichen Nutzen getroffen werden. Insofern ist eine rationale Entscheidung auf Basis dieser klinischen Evidenz nicht möglich. Solange ein Nutzenmaß über die Erstattungsfähigkeit informieren soll, gilt es, die gemessenen Effekte der patientenrelevanten Endpunkte in ein wissenschaftlich begründetes Nutzenmaß zu überführen. Um den Nutzen oder Wert einer Intervention zu analysieren, bedarf es mehrerer Wertentscheidungen. Ohne diese 
Wertentscheidungen ist es nicht möglich, einen Nutzenwert zu generieren oder eine systematische und transparente Entscheidung zu treffen.

Die Berücksichtigung der Wertvorstellungen der Versicherten und der Präferenzen der Patienten ist eine notwendige Bedingung für eine systematische und transparente Entscheidungsfindung. Es ist nicht ausreichend geklärt, auf welcher Basis bzw. welcher Evidenz die Abwägung des Nutzens und Schadens erfolgen soll. Dies ist sowohl aus der Perspektive der evidenzbasierten Medizin als auch aus Sicht der Gesundheitsökonomie eine wesentliche Lücke der Nutzenbewertung im Status Quo. Gegenwärtig werden diese Wertentscheidungen nicht transparent gemacht. Wertentscheidungen können explizit oder implizit getroffen werden. Sie können zudem auf Basis subjektiver oder objektiver Daten getroffen werden. Zunehmend wird die Forderung laut, dass implizites Wissen der Entscheidungsgremien durch explizites Wissen über diese Wertentscheidungen zu ersetzen ist. Der Anspruch an die Wissenschaftlichkeit des Bewertungsverfahrens macht eine eindeutige und klare Kommunikation der Gewichtung der Zielkriterien notwendig. Explizites Wissen kann systematisch dokumentiert und über eine wissenschaftliche Veröffentlichung der Bürger-, Versicherten- oder Patientenpräferenzen kommuniziert und korrigiert werden.

Oftmals wird in diesem Kontext darauf hingewiesen, dass das IQWiC mit der Feststellung zum Ausmaß klinischer Effektmaße betraut ist. Bei der Beurteilung der Evidenz sollten möglichst wenige Wertentscheidungen getroffen werden, da diese Aufgabe dem G-BA aufgrund seiner Legitimation vorbehalten sein sollte. Die Trennung zwischen der Informationsaufbereitung und der tatsächlichen Entscheidung, also der Trennung der Aufgaben des IQWiG und des G-BA, macht Sinn. Jedoch muss der G-BA in seiner Funktion auch in der Lage sein, auf Basis der durch das IQWiG berichteten Daten zu entscheiden. Keinen Sinn macht die Tatsache, dass der G-BA durch den Bericht des IQWiG keinerlei belastbare Informationen über die Werturteile der Versicherten bzw. Präferenzen der Patienten bekommt. In diesem Kontext müsste klar herausgestellt werden, inwiefern diese Informationen über die Präferenzen und Werturteile bereitgestellt werden bzw. wie diese Informationen für die Entscheidung des G-BA zusammenfassend beurteilt werden.

\subsection{Exkurs: FDA Pilotstudie zur Adipositaschirurgie}

Die Prävalenz von Übergewicht und Adipositas steigt weltweit. Unter den einkommensstarken Ländern haben die Vereinigten Staaten den höchsten mittleren Body-Mass-Index (BMI) bei Männern und Frauen. Mehr als zwei Drittel der über 20-Jährigen in den USA sind übergewichtig oder adipös. Übergewicht und Adipositas gehen mit einem erhöhten Risiko für Morbidität und Mortalität einher. Behandlungen für Übergewicht, mit Ausnahme der Operation, sind meist in der langfristigen Gewichtskontrolle unwirksam. ${ }^{[132]}$ Neben dem 
anhaltenden Gewichtsverlust bietet die chirurgische Behandlung zusätzliche Vorteile für die Patienten in Bezug auf Begleiterkrankungen und reduziert das relative Mortalitätsrisiko. Chang et al. ${ }^{[133]}$ kommen in ihrem Review zu dem Ergebnis, dass Adipositaschirurgie erhebliche und anhaltende positive Auswirkungen auf das Gewicht hat und adipositas-assoziierte Komorbiditäten bei der Mehrheit der übergewichtigen Patienten lindert. ${ }^{\left[{ }^{133]}\right.}$ Folglich ist die Nachfrage nach Adipositaschirurgie in den letzten Jahren gestiegen.

Dieses Thema greift auch die US Food and Drug Administration (FDA) auf. Die FDA hat eine Pilotstudie zu Patientenpräferenzen in Auftrag gegeben. Diese Studie hat zur regulatorischen Entscheidung der Agentur über die Zulassung des „Maestro Rechargeable-System“ (MP zur Behandlung von Adipositas) beigetragen. ${ }^{[132]}$ Die „Patient Preference Initiative“ untersuchte die Möglichkeiten der Integration von Patientenpräferenzen in regulatorische Entscheidungsprozesse, insbesondere die Analyse der Nutzen-Risiko-Trade offs bei MP. Zudem verfolgt das Center for Devices and Radiological Health (CDRH) das Ziel, die (wissenschaftlichen) Erkenntnisse zur Erfassung und Dokumentation von Präferenzen der Patienten und von Leistungserbringern zu erweitern. ${ }^{[334]}$ In der Pilotstudie, die in Zusammenarbeit von CDRH und RTI durchgeführt wurde, sollten Patientenpräferenzen in Bezug auf die medizintechnischen Behandlungsalternativen bei Adipositas mittels Discrete-Choice Experiment ermittelt werden. Dabei ging es um den Vergleich von Patienten, Ärzten und Entscheidern. Grundsätzlich verfolgen alle Parteien das gleiche Ziel, die Maximierung des Nutzens und die Minimierung des Schadens (bzw. des Risikos). ${ }^{[332]}$ Die Pilotstudie diente der Analyse der verschiedenen Perspektiven und der Überprüfung der Integration dieser Perspektiven und Präferenzen in die Entscheidungsprozesse im CDRH.

Die Studie schätzte die maximalen Mortalitätsrisiken, welche die Patienten bereit waren, für eine bestimmte Menge an Gewichtsverlust zu akzeptieren und die minimale Menge an Gewichtsverlust, die ausreichend ist, um die Risiken des MP zu akzeptieren. Beispielsweise, um ein Produkt mit einem Mortalitätsrisiko von $0,01 \%$ zu akzeptieren, fordert ein risikotoleranter Patient ca. 10\% Gewichtsverlust über 5 Jahre. Abbildung 5 zeigt beispielhaft die relative Gewichtung der Attribute im Discrete-Choice Experiment. ${ }^{[132]}$ Neben den klinischen Studienergebnissen berücksichtigte die FDA die Ergebnisse der Studie zur Quantifizierung der Patientenpräferenzen in Bezug auf den Nutzen und die Akzeptanz von Risiken der verschiedenen MP im Bereich der Adipositaschirurgie. Dies war das erste Mal, dass eine Patientenpräferenzstudie eine neue Gerätezulassung durch die FDA positiv beeinflusst hat. ${ }^{[32]}$ 


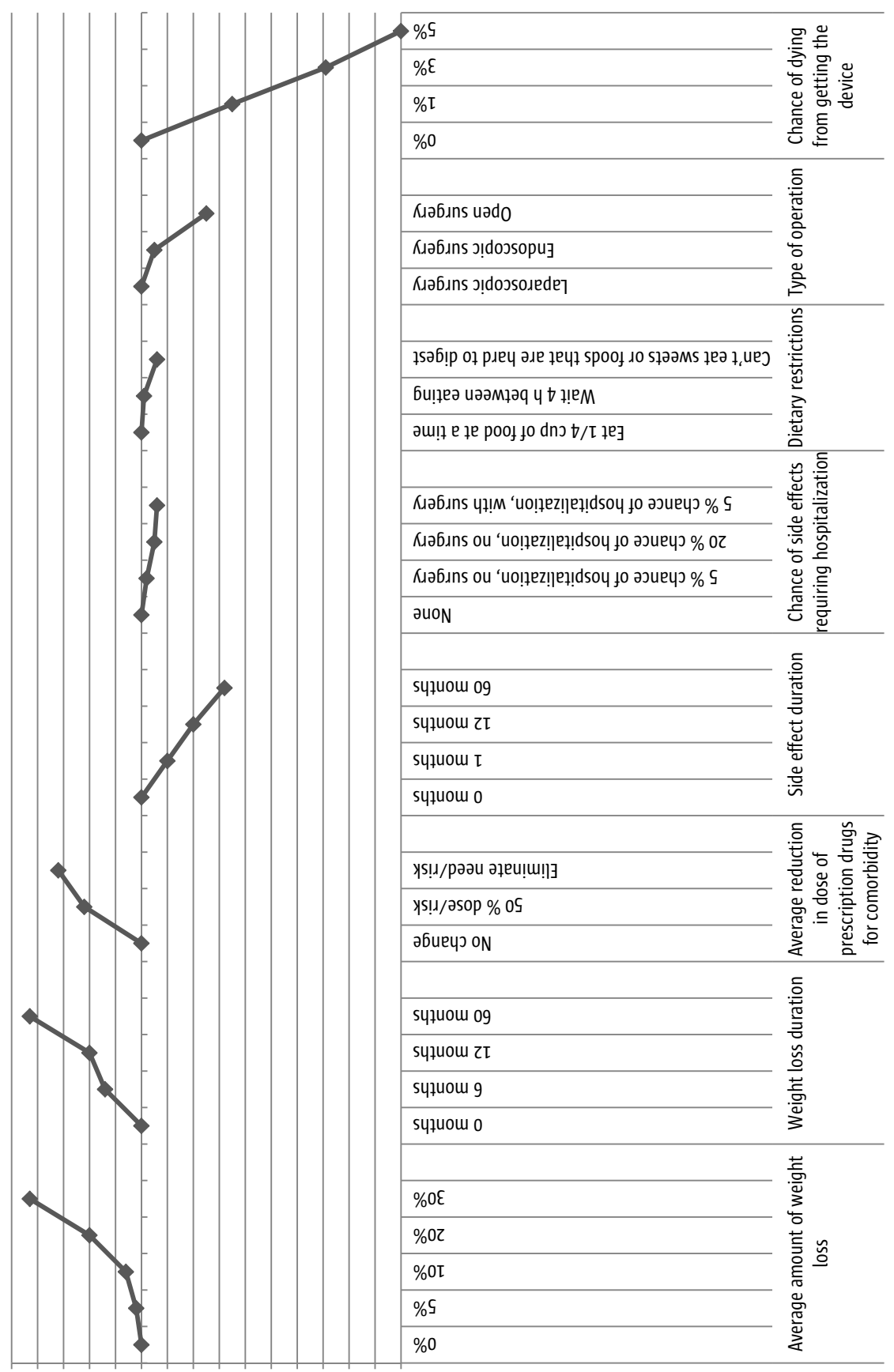

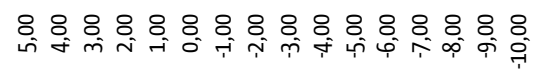

Abb. 5 Ergebnisse des DCE der Patient Preference Initiative (eigene Darstellung nach ${ }^{[132]}$ ) 


\subsection{Exkurs: Internationale Ansätze und Empfehlungen Stufenverfahren der IDEAL Collaboration zur Evaluation chirurgischer Innovationen}

2009 hat die IDEAL Collaboration ein fünfstufiges Verfahren vorgeschlagen, um für mehr Sicherheit bei der Evaluation von chirurgischen Innovationen zu sorgen. ${ }^{[19]}$ Ausgehend von der Idee und dem „Proof of concept“ sollen chirurgische und interventionelle Innovationen in mehreren Stufen weiterentwickelt, erforscht, gelernt und gelehrt werden, um sie dann systematisch zu bewerten und in der letzten Stufe in Langzeitstudien hinsichtlich ihrer Wirksamkeit zu evaluieren. ${ }^{[19]}$ (s. Tab. 4)

Daneben gibt die IDEAL Collaboration eine Reihe von Empfehlungen, wie Methodik und Berichterstattung der Forschung auf jeder dieser Stufen verbessert werden könnten. Dabei liegt der Fokus auf der Bewertung neuer invasiver Techniken und Verfahren. ${ }^{[135,136]}$

\section{Tab. 4 IDEAL Framework ${ }^{[135,136]}$}

\begin{tabular}{|c|c|}
\hline $\begin{array}{l}\text { Stufe } 1 \\
\text { „IDEE“ }\end{array}$ & $\begin{array}{l}\text { Initialer Bericht } \\
\text { - Innovation kann geplant, zufällig oder erzwungen sein } \\
\text { - Fokus auf Erläuterung und Beschreibung }\end{array}$ \\
\hline $\begin{array}{l}\text { Stufe } 2 \text { a } \\
\text { „ENTWICKLUNG“ }\end{array}$ & $\begin{array}{l}\text { "Basteln“ (schnelle iterative Modifikation der Technik und Indikationen) } \\
\text { - Geringe Erfahrungen von einem Zentrum } \\
\text { - Fokus auf technische Details und Durchführbarkeit }\end{array}$ \\
\hline $\begin{array}{l}\text { Stufe } 2 b \\
\text { „EXPLORATION“ }\end{array}$ & $\begin{array}{l}\text { - Technik stabil und erprobt } \\
\text { - Replikation durch andere } \\
\text { - Fokus auf Nebenwirkungen und potenziellem Nutzen } \\
\text { - Lernkurven wichtig } \\
\text { - Definition und Entwicklung von Qualitäts-parametern }\end{array}$ \\
\hline $\begin{array}{l}\text { Stufe } 3 \\
\text { „BEWERTUNG“ }\end{array}$ & $\begin{array}{l}\text { - Gewinnt breite Akzeptanz } \\
\text { - Wird als möglicher Ersatz für aktuelle Therapie angesehen } \\
\text { - Vergleich gegen aktuellen Best-Practice }\end{array}$ \\
\hline $\begin{array}{l}\text { Stufe } 4 \\
\text { „LANGZEIT MONITORING“ }\end{array}$ & $\begin{array}{l}\text { - Überwachung später und seltener Probleme } \\
\text { - Veränderungen in der Anwendung }\end{array}$ \\
\hline
\end{tabular}

Stufe 1 der Innovation beginnt, wenn ein Chirurg oder eine kleine Gruppe von Chirurgen eine innovative Prozedur zum ersten Mal anwenden. Wenn erste Berichte einen Nutzen andeuten, können einige „Early Adopters“ die Innovation aufnehmen (Stufe 2a). In dieser Phase (Entwicklungsphase) liegt der Fokus auf der technischen Entwicklung des Verfahrens. Anschließend liegt die Aufmerksamkeit auf der Untersuchung der Indikationen, in denen das Verfahren verwendet wird, dem Verständnis des potenziellen Nutzens und Schadens sowie der Erhöhung der Wirksamkeit der Innovation (Stufe 2b, Explora- 
tionsphase). Early Adopters verfeinern in dieser Zeit ihre Fähigkeiten, verbessern ihre Lernkurve. Um diese Ziele zu erreichen, sind für die IDEAL Collaboration prospektive Forschungsdatenbanken wertvoll. Diese sorgfältig geplanten, prospektiven, unkontrollierten klinischen Studien könnten als parallele Ergänzungen zu kleineren Machbarkeits- oder explorativen randomisierten klinischen Studien durchgeführt werden. Genau charakterisierte und relevante Zielkriterien sind dabei sowohl für Forschungsdatenbanken und randomisierte Studien entscheidend. Diese sollten technische, klinische und patientenrelevante Outcomes beinhalten. ${ }^{[135,136]}$

Während die ersten beiden Stufen auf die Entwicklung einer neuen Technologie und die Beschreibung ihrer Outcomes ausgerichtet sind, soll die dritte Stufe die Wirksamkeit gegenüber geltenden Standards bewerten. Auf der Stufe 3 ist die entscheidende Frage, ob die Innovation besser als etablierte Verfahren in Bezug auf die klinische Wirksamkeit und Wirtschaftlichkeit ist (Bewertungsphase). Wenn die Möglichkeit zur robusten Evaluation nicht ergriffen wird, kann eine weit gefasste Adoption der Innovation ohne ausreichende Beweise geschehen.

Gemäß der IDEAL Collaboration sollten RCT der Standard in dieser Phase sein. Dennoch geht auch die Gemeinschaft davon aus, dass RCT mit chirurgischen Techniken manchmal unnötig oder nicht möglich sind oder Anpassungen bzw. zusätzliche Funktionen benötigen. In diesem Fällen empfiehlt die IDEAL Collaboration alternative Studiendesigns, wie nicht-randomisierte Parallelgruppenstudien, kontrollierte unterbrochene Zeitreihen, Keil-/Stufendesigns (randomisierte Roll-outs), Tracker-Studien oder Kenntnis-basierte randomisierte Studien. ${ }^{[135]}$

Schließlich wird in Stufe 4, wenn die Innovation weithin akzeptiert und eingesetzt ist, deren Wirksamkeit bei der Routineanwendung überwacht (Phase der Langzeitstudien). Seltene Ereignisse und langfristigen Ergebnisse könnten in dieser Phase deutlich werden und die Ergebnisvariabilität kann zur Klärung der Indikationen oder wichtiger technischer Details führen. Die Ergebnisse der Studie können auf die Routinepraxis verallgemeinert und Indikationen erweitert werden. Das typische Studiendesign dafür sind Register zur Überwachung der neuen Technik sowie der Indikationen. ${ }^{[135]}$

\section{EAES Empfehlungen zum Innovationsmanagement in der Chirurgie}

Im Rahmen eines Mandats der Europäischen Vereinigung für die endoskopische Chirurgie (EAES) wurde 2010 ein Leitfaden zur Methodik des Innovationsmanagements in der endoskopischen Chirurgie entwickelt. ${ }^{[137]}$ Der Schwerpunkt dieser Richtlinie liegt auf Patientensicherheit, Wirksamkeit und Effizienz und dient der Entwicklung von Empfehlungen für die Bewertung und Einführung von chirurgischen Innovationen. Die Entwicklung und Umset- 
zung von Innovationen in der Chirurgie ist demnach in fünf Abschnitte gegliedert:

1. Definition von Innovation,

2. präklinische wissenschaftliche Entwicklung,

3. klinische wissenschaftliche Entwicklung,

4. wissenschaftliche Zustimmung/Zulassung,

5. die Umsetzung in der Praxis zusammen mit der Überwachung.

In Bezug auf die präklinische Entwicklung neuer, innovativer Technologien geht die Empfehlung davon aus, dass eine klinische Bewertung/Studie initiiert werden muss, wenn nicht genügend klinische Evidenz zu Sicherheit und Wirksamkeit vorliegt. Um angemessene Evidenz zu erreichen, muss dafür jedoch „nicht unbedingt eine randomisierte kontrollierte Studie““[37] erfolgen . Vielmehr müssten bei der Initiierung einer Studie mehrere Faktoren berücksichtigt werden: die Lern- und Kompetenzkurve, die Identifizierung von Störfaktoren, um systematische Verzerrung zu minimieren, die Ausbildungsanforderungen sowie Anforderungen des Forschers (Studienzentrums). Die entsprechende Auswertung hängt hauptsächlich von der Art und der Komplexität der Innovation ab.

Die EAES erkennt an, dass RCT nicht immer realisierbar, möglich oder angemessen sind ${ }^{[106]}$ und empfiehlt daher alternative Designs, wie „parallel group non-randomized studies“ oder „controlled interrupted time-series“"[135] oder prospektive Kohortenstudien. ${ }^{[137]}$ Nachdem Informationen über klinische, wirtschaftliche, ethische, soziale und rechtliche Aspekte gesammelt wurden, sollte ein HTA durchgeführt werden. Innovationen in der klinischen Praxis sollten mit einem möglichst hohen Grad an Sicherheit für den Patienten eingeführt werden (nicht schaden). Die Empfehlungen der EAES dienen der Erreichung dieses Ziels ohne zukünftige vielversprechende diagnostische und therapeutische Innovationen zu verhindern.

\section{Zulassung- und Nutzenbewertungsverfahren in den USA - „Pre-Market Approval“ der FDA}

Die Zulassung von MP in den USA erfolgt durch die U.S. Food and Drug Administration. Die FDA ist dafür verantwortlich, die Sicherheit und Wirksamkeit der MP festzustellen, bevor diese auf dem Markt vertrieben werden können. Die USA besitzen, ähnlich wie Europa, hierzu ebenfalls ein Klassifikationssystem, welches die MP in drei Risikogruppen unterteilt. ${ }^{[41]}$ Die Zulassung von MP erfolgt hauptsächlich durch zwei Verfahren: die ,Premarket Notification“ (PMN) sowie die ,Premarket Approval' (PMA). Aber auch durch andere Verfah-

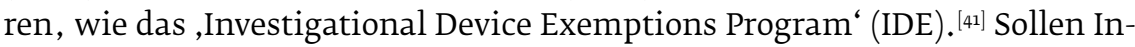
novationen zugelassen werden, ist im Rahmen der ,Premarket Approval'-Zulassung die Wirksamkeit des Produktes in Form von Studien nachzuweisen. Diese Art von Zulassung ist für alle Produkte der Klasse III prinzipiell verpflich- 
tend. Ebenfalls muss dieser Prozess für Produkte der Klassen I und II durchgeführt werden, die keine Ähnlichkeiten zu einem ,Predicate Device“ (Vorgängermodell) aufweisen. ${ }^{[138]}$

Die Sicherheits- und Wirksamkeitsüberprüfung der MP erfolgt in erster Linie durch das ,Medical Device Reporting“-Programm sowie das ,Postmarketing Surveillance Studies'-Programm. Die Kontrollen können aber auch in Form von epidemiologischen Untersuchungen sowie in Form von Begehungen der Hersteller erfolgen. ${ }^{[4]}$ Vom Hersteller werden Meldungen an die FDA über sogenannte Vorkommnisse (mit einem bestimmten Schweregrad) mit ihren MP erwartet. Leistungsanbieter, z.B. Krankenhäuser, ambulante Einrichtungen, Pflegeheime, sollen ernsthafte Vorfälle an die Hersteller und Todesfälle, die u.a. durch das MP verursacht wurden, an den Hersteller und die FDA melden. ${ }^{\left[{ }^{41}\right]}$ In den USA werden die Erstattungen für MP durch das ,Centers for Medicare \& Medicaid Services' (CMS) sowie das ,Veteran Affairs Health System“ für die stattlichen Krankenversicherungen geregelt. Die privaten Krankenversicherungen legen die Erstattungen selber fest, orientieren sich aber dabei an die Entscheidungen des CMS. ${ }^{[138]}$ Die Erstattungen im stationären und ambulanten Bereich durch Medicare werden im „Social Security Act“ definiert. Um erstattungsfähig zu sein, muss ein Produkt als ,Reasonable and Necessary“, also als angemessen und notwendig, bewertet werden. ${ }^{[138]}$

Damit innovative Produkte schneller für den Markt zugelassen werden, wurde ein Pilotprojekt gestartet, um den Zeitraum zwischen der Marktzulassung durch die FDA und die Erstattung durch die CMS zu verkürzen. Dabei sollen durch die gleichzeitige Überprüfung von Zulassungsanträgen pro Jahr fünf MP parallel bewertet werden. ${ }^{[138]}$ Eine kurze Gegenüberstellung der Zulassungsund Nutzenbewertungsverfahren der EU im Vergleich zu den USA findet sich in Abbildung 6.

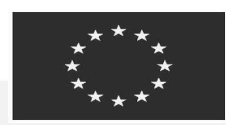

- Prüfung dezentral durch privatwirtschaftliche "benannte Stellen“

- Klinische Studien nur für implantierbare Medizinprodukte und Produkte der Risikoklasse III grundsätzlich notwendig

- Nachweis von Sicherheit und Leistungsfähigkeit

- Zulassung i.d.R. ohne Auflagen

- Dezentrale behördliche Überwachung nur in besonderen Fällen

- Daten zur Sicherheit und Wirksamkeit nicht öffentlich zugänglich

- Zulassung durch FDA

- Klinische Studien grundsätzlich notwendig

- Nachweis von Sicherheit und Leistungsfähigkeit

- Zulassung häufig mit Auflagen

- Zentrale behördliche Überwachung des Marktes ist die Regel, insb. bei Interventionen

- Daten zur Sicherheit und Wirksamkeit öffentlich im Internet zugänglich

Abb. 6 Zulassungsverfahren im Vergleich: EU \& USA (eigene Darstellung in Anlehnung an ${ }^{[5]}$ ) 


\section{Medical Device Innovation Consortium (MDIC) - Patient Centered Benefit-Risk Project}

Das „Medical Device Innovation Consortium“ (MDIC) zielt durch seine Publicprivate-Partnership darauf ab, regulatorische Entscheidungen in der Medizinprodukte-Industrie voranzutreiben. Das MDIC ist die einzige öffentlich-private Partnerschaft, die ausschließlich die strategischen Anforderungen der Medizinprodukte-Industrie fokussiert. MDIC wurde entwickelt, um eine kollaborative Umgebung zu schaffen, in der Industrie, gemeinnützige Organisationen und Regierungen zusammenarbeiten können, um vorwettbewerbliche Forschung in der Medizintechnik voranzutreiben. Dadurch sollten die Hersteller den Bedürfnissen der Patienten in den USA rechtzeitiger begegnen können. MDIC schafft einen Ausgleich zwischen den Bedürfnisse der Medizinprodukte-Industrie, der FDA, und den damit verbundenen Organisationen und den Bedürfnissen der Bevölkerung der Vereinigten Staaten.

Die FDA hat Medizintechnikunternehmen aufgefordert, die Patientenperspektive in Bezug auf die Vorteile und Risiken eines neuen MP zu berücksichtigen, wenn sie die Marktzulassung anstreben. Im Rahmen eines Projektes hat die MDIC die Grundlagen für eine patientenzentrierte Nutzen-Risikoabwägung analysiert. Anfang Mai 2015 wurde der Projektbericht „Medical Device Innovation Consortium (MDIC) - Patient Centered Benefit-Risk Project“ veröffentlicht. Dieser gibt Einblicke in die ersten Ergebnisse des Projektes. ${ }^{[39]}$

Da es keine allgemein anerkannten Konzepte für die Integration von Patientenpräferenzen in die Zulassungsverfahren gibt und diese Informationen daher bisher kaum verwendet wurde, soll dieser MDIC-Rahmenbericht das Verständnis der Industrie, der FDA-Mitarbeiter und anderer verbessern, wie die Perspektive der Patienten in das behördliche Zulassungsverfahren integriert werden könnte. Der Bericht liefert Hintergrundinformationen über die Konzepte der Nutzen-Risiko-Bewertung sowie der Patientenpräferenzen; erörtert den potenziellen Wert der Integration von Nutzen-Risiko-Abwägungen in einen Zulassungsantrag; diskutiert, wann im Produktlebenszyklus derartige Informationen gesammelt werden könnten und beschreibt Faktoren, die bei der Auswahl einer Präferenzmessmethode berücksichtigt werden müssen. Zudem werden Überlegungen hinsichtlich der Verwendung von Informationen zu Patientenpräferenzen in den Regulierungsprozess sowie deren Einsatzmöglichkeiten in der Erstattungsentscheidung, dem Marketing und der gemeinsamen medizinischen/therapeutischen Entscheidungsfindung diskutiert.

Der Abschnitt „Mögliche Nutzung und Wert von Informationen zu Patientenpräferenzen im Produktentwicklungszyklus“ des Berichts beschreibt und diskutiert die drei wichtigsten Verwendungen von Präferenzinformationen:

1. Nutzen-Risiko-Abwägungen,

2. Identifikation von Patientengruppen, die die Verwendung einer bestimmten Technologie präferieren würden und 
3. die Bereitstellung der erforderlichen Informationen, um ein quantitatives Nutzen-Risiko-Modell zu erstellen.

Das Kapitel stellt dann das Konzept des Produktentwicklungszyklus' vor und diskutiert, wie Informationen zu Patientenpräferenzen in jeder Phase des Lebenszyklus' nützlich sind und in welchem Schritt des Produktlebenszyklus' es hilfreich sein kann, derartige Informationen zu sammeln. ${ }^{[139]}$

\section{Patient Preference Information - Submission, Review in PMAs, HDE Applications, and De Novo Requests, and Inclusion in Device Labeling}

Die FDA-Richtlinie zu „Faktoren, die bei der Erstellung von Nutzen-Risiko-Ermittlungen in Medical Device Premarket Approval und De Novo Klassifikation“ (auch „Benefit-Risk Guidance“ genannt) erklärt, dass verschiedene Patientenperspektiven in der Datenerhebung während des vorbehördlichen Review-Prozess für PMA-Anträge und De Novo-Klassifizierungsanfragen betrachten sollten. Diese Leitlinie legt fest, dass die Risikobereitschaft der Patienten und ihre Perspektive auf Nutzen und Vorteile einer Technologie, zusätzlich zu verschiedenen anderen Faktoren, in der FDA-Bewertung des Nutzen-Risiko-Profils von bestimmten MP berücksichtigt werden sollte. Allerdings müssen die Informationen die Standards der FDA für gültige wissenschaftliche Erkenntnisse erfüllen.

Am 18. Mai 2015 veröffentlichte die FDA einen weiteren Richtlinienentwurf $\mathrm{zu}$ „Patient Preference Information - Submission, Review in PMAs, HDE Applications, and De Novo Requests, and Inclusion in Device Labeling “.."140] Dieser Entwurf ergänzt die vorherige Richtlinie und gibt Leitlinien zum Umgang mit Informationen über Patientenpräferenzen, die von der FDA in der Entscheidungsfindung in Bezug auf PMAs, Humanitarian Device Exemption (HDE)Anwendungen und De Novo-Anfragen verwendet werden können. Die Ziele dieses Entwurfs der Leitlinien sind:

1. die Förderung der freiwilligen Vorlage von Daten zu Patientenpräferenzen durch die Hersteller oder andere Beteiligte in bestimmten Fällen

2. die Darstellung von empfohlenen Qualitäten der Daten zu Patientenpräferenzen, die in validen wissenschaftlichen Erkenntnissen resultieren können

3. Bereitstellung von Empfehlungen für die Erhebung von Daten zu Patientenpräferenzen für die FDA

4. die Bereitstellung von Empfehlungen für die Integration von Daten zu Patientenpräferenzen in der Kennzeichnung/Information für Patienten und Anwender.

Der Richtlinienentwurf enthält zudem einige hypothetische Beispiele, wie Präferenzdaten in regulatorischen Entscheidungen der FDA integriert werden können. 
Die Einreichung von Daten zu Patientenpräferenzen bei der FDA ist freiwillig. Allerdings können diese Präferenzdaten für die FDA während der Nutzen-Risiko-Abwägung für MP auf entscheidende Art und Weise nützlich sein:

1. Identifizierung der wichtigsten Vorteile und Risiken einer Technologie aus der Sicht des Patienten

2. Bewertung der relativen Bedeutung verschiedener Attribute von Nutzen und Risiken für die Patienten und Klärung, wie Patienten zwischen diesen Vorteilen und Risiken für eine bestimmte Technologie abwägen

3. besseres Verständnis der Heterogenität oder der Verteilung von Patientenpräferenzen hinsichtlich Nutzen und Risiken verschiedener Behandlungs- oder Diagnosemöglichkeiten.

Da der Wirkmechanismus für MP oft gut charakterisiert und lokalisiert werden kann, geht der FDA-Entwurf davon aus, dass Patientenpräferenzen für MP einfacher zu erheben sind als für pharmazeutische oder biologische Therapien. Bei diesen würden mehrheitlich systemische Wirkungen auftreten und Off-Target Nebenwirkungen nicht immer umfassend vorab antizipiert werden können. ${ }^{[140]}$ 